\title{
Association of HMGB1, BRCA1 and P62 expression in ovarian cancer and chemotherapy sensitivity
}

\author{
SHOUYONG $\mathrm{LI}^{1 *}$ and YANLEI WEI ${ }^{2 *}$ \\ Departments of ${ }^{1}$ Clinical Laboratory and ${ }^{2}$ Pharmacy, Dongying People's Hospital, \\ Dongying, Shandong 257091, P.R. China
}

Received August 7, 2017; Accepted January 2, 2018

DOI: $10.3892 / \mathrm{ol} .2018 .8482$

\begin{abstract}
The expression of high mobility group box 1 (HMGB1), breast cancer susceptibility gene 1 (BRCA1) and $P 62$ in ovarian cancer was investigated to explore its association with chemotherapy sensitivity in ovarian cancer patients. Tumor tissues and para-carcinoma normal tissues of 60 ovarian cancer patients hospitalized in Department of Surgery in Dongying Hospital from June, 2012 to June, 2015 were collected. Reverse transcription-quantitative polymerase chain reaction (RT-qPCR) was used to detect the mRNA expression levels of HMGB1, BRCA1 and P62 in tumor and para-carcinoma normal tissues. Moreover, immunohistochemistry was used to detect the protein expression of HMGB1, BRCA1 and P62 in tumor tissues and para-carcinoma normal tissues. The cancer tissue specimens were divided into the chemotherapy resistance group and sensitivity group through the in vitro resin droplet experiment to analyze the association of the expression of HMGB1, BRCA1 and P62 in epithelial ovarian cancer with chemotherapy resistance of patients. The RT-qPCR results showed that the expression of HMGB1, BRCA1 and P62 in ovarian cancer tissues at the mRNA level was significantly higher than that in para-carcinoma normal tissues. Immunohistochemical results showed that the positive expression levels of HMGB1, BRCA1 and P62 in ovarian carcinoma tissue were $61.67 \%$ (37/60), 76.33\% (47/60) and 71.67\% (43/60), respectively, while the positive expression levels of HMGB1, BRCA1 and P62 in para-carcinoma normal tissues were $13.33 \%$ (8/60), $8.33 \%$ $(5 / 60)$ and $11.67 \%(7 / 60)$, respectively, and the differences were statistically significant $(\mathrm{P}<0.05)$. In vitro resin droplet
\end{abstract}

Correspondence to: Dr Shouyong Li, Department of Clinical Laboratory, Dongying People's Hospital, 1216 First South Road, Dongying, Shandong 257091, P.R. China

E-mail: lishouyongdy@163.com

*Contributed equally

Key words: ovarian cancer, high mobility group box 1, breast cancer susceptibility gene 1, P62, chemotherapy sensitivity experiment revealed that 38 out of 60 ovarian cancer patients were drug resistant and 22 patients were sensitive to the therapy. The analysis of the association with chemotherapy sensitivity revealed that the positive expression of HMGB1, BRCA1 and P62 was associated with the drug resistance of ovarian cancer patients. The positive expression of HMGB1, BRCA1 and P62 was associated with chemotherapy sensitivity of ovarian cancer patients. Therefore, HMGB1, BRCA1 and P62 may be molecular markers for the prediction of chemotherapy sensitivity of ovarian cancer patients.

\section{Introduction}

Ovarian cancer is a common malignant tumor in women and its morbidity and mortality are high (1). At present, the main therapeutic regime is surgical therapy first, followed by chemotherapy using platinum drugs. Previous findings showed that chemotherapy can prolong the lifetime of patients (2). Multi-drug resistance occurs in approximately $25 \%$ of ovarian cancer patients, which leads to the decreased curative effect of chemotherapy, thus reducing the survival rate and quality of life of patients (3).

High mobility group box 1 (HMGB1) is a non-histone nuclear protein that exists in nucleus extensively. At the same time, a small amount of HMGB1 is present in the cytoplasm and cytomembrane (4). HMGB1 is known to play a key role in the occurrence, progression, metastasis and drug resistance of tumors (5). Breast cancer susceptibility gene 1 (BRCA1) is a kind of repair gene for DNA damage, and can lead to the drug resistance of tumor cells by repairing the cell damage caused by chemotherapy drugs $(6,7)$. Clinical studies found that the sensitivity to cisplatin chemotherapy and the prognosis of patients with a specifically high expression of BRCAl gene are worse than those of patients with a specifically low expression of $B R C A 1$ gene (8). P62, a multi-functional protein, is abnormally expressed in most tumors and play a key role in proliferation, differentiation, anti-apoptosis and induced autophagy (9).

In order to analyze the expression of HMGB1, BRCA1 and P62 in ovarian carcinoma tissues and the association with chemotherapy sensitivity of ovarian cancer patients, reverse transcription-quantitative polymerase chain reaction (RT-qPCR) and immunohistochemistry were used to investigate the expression of HMGB1, BRCA1 and P62 in 
tumor and para-carcinoma normal tissues and analyze the association with chemotherapy sensitivity of cisplatin in order to provide a research basis for clinical rational drug use.

\section{Materials and methods}

Materials. In the present study, tumor tissues and the corresponding para-carcinoma normal tissues of 60 ovarian cancer patients admitted into the Department of Surgery in Dongying Hospital (Dongying, China) from June, 2012 to June, 2015, were selected. The patients were definitively diagnosed with ovarian cancer by clinical pathology and did not receive chemotherapy and surgical therapy. Patients were aged $24-78$ years, and the median age was 52 years. This study was approved by the Clinical Ethics Committee of Dongying People's Hospital. At the same time, all the patients enrolled signed the informed consent form.

RNA extraction, reverse transcription, and RT-qPCR kit (all from Invitrogen; Thermo Fisher Scientific, Inc., Waltham, MA, USA), HMGB1, BRCA1, P62, GAPDH antibodies (1:800; cat. nos. 10829-1-AP, 22362-1-AP, 18420-1-AP, 10494-1-AP) and HRP-labeled secondary antibodies (1:1000; cat. no. SA00001-2) (all from Proteintech Biotechnology Co., Ltd.; Wuhan Sanying Biotechnology, Wuhan, China); immunohistochemical staining kit SP-9001 (Beijing Zhongshan Goldenbridge Biotechnology Co., Ltd.; OriGene Technologies, Inc., Beijing, China), primer synthesis (Takara Biotechnology Co., Ltd., Dalian, China) were used in the present study.

Detection of the $m R N A$ expression of HMGB1, BRCA1 and P62 in tissue specimens of patients via RT-qPCR. Approximately $100 \mathrm{mg}$ tumor and para-carcinoma normal tissues of patients frozen in liquid nitrogen were taken and the total RNA was extracted according to the instructions of the RNA extraction kit. An ultraviolet and visible spectrophotometer (Hitachi Ltd., Tokyo, Japan) was used to detect the absorbance value of the total RNA extracted at 260 and $280 \mathrm{~nm}$, and the total RNA samples with the A260/A280 value within 1.8-2.0 were selected for the follow-up experiment.

The reverse transcription reaction was carried out according to the instructions of the reverse transcription kit, and the cDNA obtained served as the template. The mRNA expression of HMGB1, BRCA1 and P62 was detected according to the instructions of the PCR kit with GAPDH as the control gene. The primer sequences are shown in Table I. The reaction conditions were: $95^{\circ} \mathrm{C}$ for $10 \mathrm{~min}, 95^{\circ} \mathrm{C}$ for $15 \mathrm{sec}, 60^{\circ} \mathrm{C}$ for $30 \mathrm{sec}, 72^{\circ} \mathrm{C}$ for $30 \mathrm{sec}$, a total of 42 cycles for amplification; $72^{\circ} \mathrm{C}$ for $5 \mathrm{~min}$. The $\mathrm{C}_{\mathrm{q}}$ value was read, and the $2^{-\Delta \mathrm{Cq}}$ method was used to calculate the relative expression level. The formula used was: $\Delta \mathrm{C}_{\mathrm{q}}$ (target gene) $=\mathrm{C}_{\mathrm{q}}$ (target gene) $-\mathrm{C}_{\mathrm{q}}$ (control gene).

Detection of the protein expression of $H M G B 1, B R C A 1$ and P62 in tissue specimens of patients via immunohistochemistry. After the tissues were obtained during surgery, they were fixed using $4 \%$ formalin, and then embedded in paraffin, followed by conventional sections. The operation was carried out according to the instructions of the SP-9001 immuno-histochemical kit. The paraffin sections were dewaxed and hydrated for pretreatment, and incubated at $3 \% \mathrm{H}_{2} \mathrm{O}_{2}$ at room temperature
Table I. RT-qPCR primer sequence.

\begin{tabular}{ll}
\hline Genes & \multicolumn{1}{c}{ Primer sequence } \\
\hline HMGB1 & F 5'-TGGACTGCTCAGGAAAC-3' \\
& R 5'-AGGGGCAAACCGTAAT-3' \\
BRCA1 & F 5'-CCCATTTTCCTCCCGCA-3' \\
& R 5'-GGACCTTGGTGGTTTCTTCCA-3' \\
P62 & F 5'-CCAGCACCAAGAGCACGGACAGCG-3' \\
& R 5'-TGGGGAGAAGAAGGGGACCACGAA-3' \\
GAPDH & F 5'-ATGGCACCGTCAAGGCTGAG-3' \\
& R 5'-GCAGTGATGGCATGGACTGT-3'
\end{tabular}

$H M G B 1$, high mobility group box $1 ; B R C A 1$, breast cancer susceptibility gene 1; GAPDH, glyceraldehyde 3-phosphate dehydrogenase; RT-qPCR, reverse transcription-quantitative polymerase chain reaction; F, forward; R, reverse.

for $10 \mathrm{~min}$. Then they were heated and repaired by citric acid buffer and sealed using 10\% goat serum. The primary antibody (dilution 1:100) was added for incubation at $4^{\circ} \mathrm{C}$ overnight. After the sections were washed with phosphate-buffered saline (PBS), the biotin-labeled secondary antibody was added for incubation at room temperature for $20 \mathrm{~min}$, followed by washing using PBS, color development via diaminobenzidine, hematoxylin staining, dehydration, transparency via xylene, sealing via neutral resin, and microscopic image (TE2000-U; Nikon Instruments Europe BV, Amsterdam, The Netherlands).

Five fields (x400) were selected randomly in each section. The staining results were scored according to the staining intensity and the percentage of positive cells: The number of positive cells were scored as: $<5 \%$ : 0 point; $5-25 \%$ : 1 point; 26-50\%: 2 points; >50\%: 3 points. The staining intensity was scored as: No staining: 0 point; light yellow: 1 point; brown yellow: 2 points; dark brown: 3 points. Both scores above were added: $\leq 3$ points indicated a negative expression, while $>3$ points indicated a positive expression, and the statistical results were analyzed.

Detection of patient's drug resistance to cisplatin via in vitro resin droplet experiment. Tumor tissues of the patients were obtained during surgery, immediately digested using the digestive enzyme, filtered and centrifuged to collect cells. RPMI-1640 culture medium containing $10 \%$ fetal calf serum was added to prepare the cell suspension. Cell-collagen mixture was prepared in an ice bath, then inoculated onto a 24-well plate and cultured for $24 \mathrm{~h}$. The cells were divided into the administration group (cisplatin) and blank group (no drug) and cultured for $24 \mathrm{~h}$. Then neutral red solution was added and cells were fixed with $10 \%$ formaldehyde solution. The in vitro drug sensitivity was evaluated by calculating the number of cells in the administration group/the number of cells in blank group, with $<50 \%$ indicating drug sensitivity, and $\geq 50 \%$ indicating cell drug resistance (10).

Association of the expression of HMGB1, BRCA1 and P62 with drug resistance of patients. According to the expression 
A

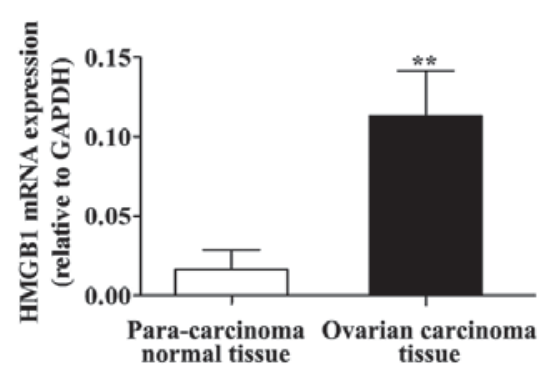

B

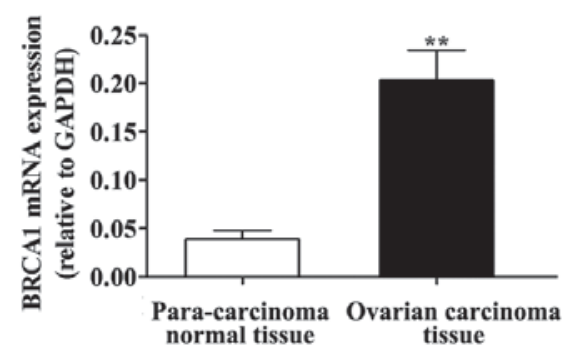

C

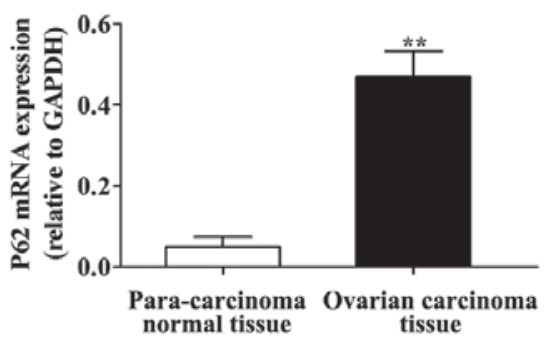

Figure 1. Detection of the mRNA expression of HMGB1, BRCA1 and P62 via RT-qPCR. mRNA expression of (A) HMGB1, (B) BRCA1, and (C) P62 in tissue specimens. Compared with those in para-carcinoma normal tissue, the mRNA expression levels of HMGB1, BRCA1 and P62 are significantly higher in ovarian carcinoma tissue, ${ }^{* *} \mathrm{P}<0.01$. HMGB1, high mobility group box 1; BRCA1, breast cancer susceptibility gene 1; RT-qPCR, reverse transcription-quantitative polymerase chain reaction.
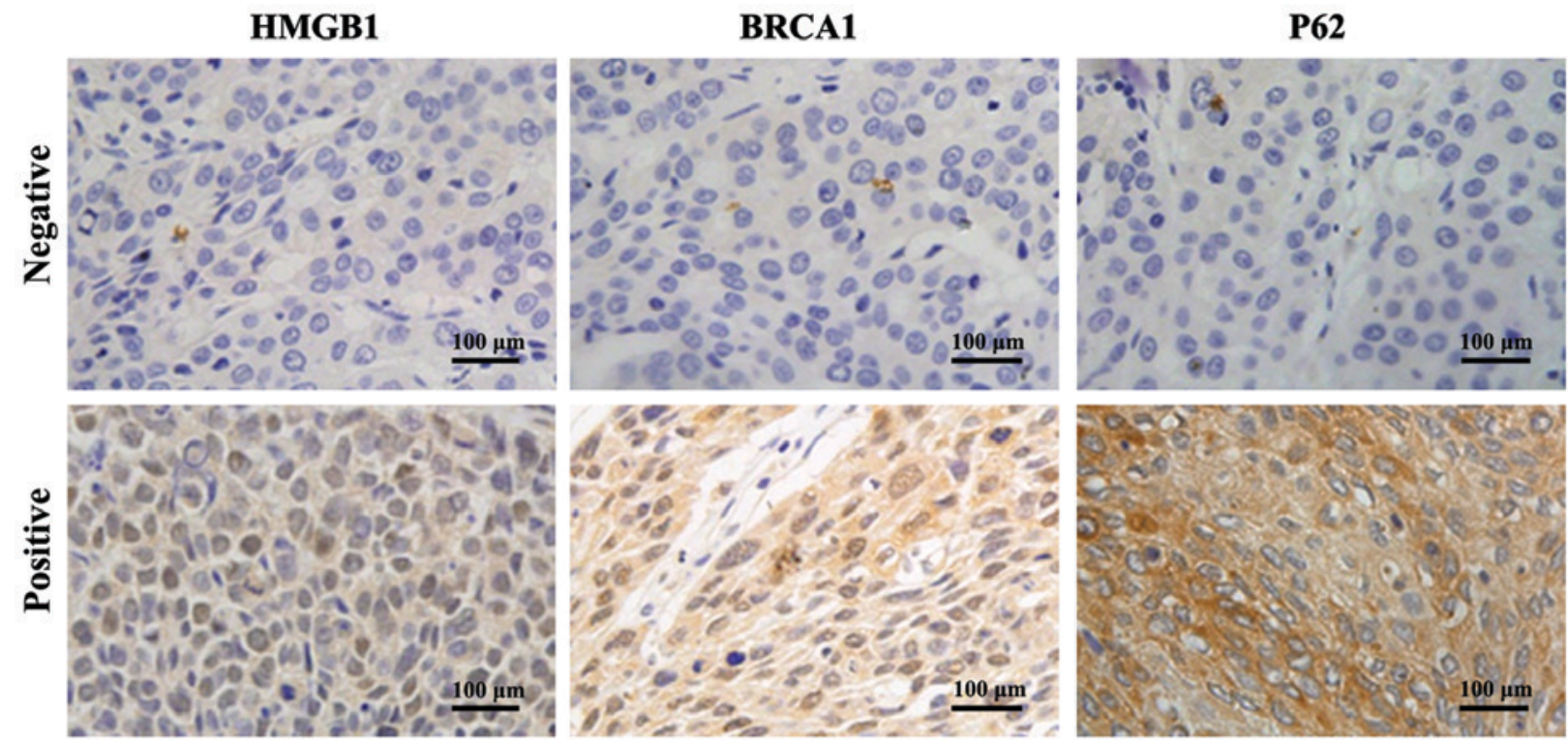

Figure 2. Detection of protein expression of HMGB1, BRCA1 and P62 in tissues of clinical patients (x400) via immunohistochemistry. Positive immunohistochemical staining of HMGB1, BRCA1 and P62 are all yellow brown; HMGB1 protein is mainly present in cytoplasm; BRCA1 mainly exists in cell nucleus, and partially in cytoplasm; and P62 mainly exists in cytoplasm. HMGB1, high mobility group box 1; BRCA1, breast cancer susceptibility gene 1.

status of HMGB1, BRCA1 and P62 in ovarian carcinoma tissue, 60 ovarian cancer patients were divided into the positive and negative expression groups. A Chi-square test was used to analyze the association of the expression of HMGB1, BRCA1 and P62 in ovarian carcinoma tissue with the drug resistance of patients to cisplatin.

Statistical analysis. SPSS 17.0 software (SPSS, Inc., Chicago, IL, USA) was used for data processing. Measurement data were expressed as mean \pm standard deviation. A t-test was used for intergroup comparison, the Chi-square test was used for intergroup comparisons of enumeration data. $\mathrm{P}<0.05$ was considered to indicate a statistically significant difference.

\section{Results}

Detection of the MRNA expression of HMGBI, BRCAI and P62 in tissue specimens via RT-qPCR. The RT-qPCR detection results are shown in Fig. 1. Compared with those in para-carcinoma normal tissues, the mRNA expression of
HMGB1, BRCA1 and P62 was significantly higher in ovarian carcinoma tissues, and the differences were statistically significant $(\mathrm{P}<0.01)$.

Detection of the protein expression of HMGB1, BRCAl and P62 in tissue specimens by immunohistochemistry. Immunohistochemistry detection results are shown in Fig. 2. The positive immunohistochemical staining of HMGB1, BRCA1 and P62 were all yellow brown, with HMGB1 protein mainly being identified in the cytoplasm, BRCA1 mainly in the cell nucleus, and partially in the cytoplasm; and P62 mainly being identified in the cytoplasm.

The scores of statistical staining are shown in Table II. The positive expression rates of HMGB1 in ovarian carcinoma and para-carcinoma normal tissues were 61.67\% (37/60) and $13.33 \%(8 / 60)$, and the difference was statistically significant $(\mathrm{P}<0.01)$; the positive expression rates of BRCA1 in ovarian carcinoma and para-carcinoma normal tissues were $78.33 \%$ (47/60) and 8.33\% (5/60), and the difference was statistically significant $(\mathrm{P}<0.01)$. The positive expression rates of $\mathrm{P} 62$ in 
Table II. Protein expression of HMGB1, BRCA1 and P62 in para-carcinoma normal tissues and ovarian carcinoma tissues.

\begin{tabular}{|c|c|c|c|c|c|c|c|c|c|c|}
\hline \multirow[b]{2}{*}{ Groups } & \multirow[b]{2}{*}{ No. } & \multicolumn{3}{|c|}{ HMGB1 } & \multicolumn{3}{|c|}{ BRCA1 } & \multicolumn{3}{|c|}{ P62 } \\
\hline & & Positive & $\begin{array}{l}\text { Positive } \\
\text { rate }\end{array}$ & P-value & Positive & $\begin{array}{l}\text { Positive } \\
\text { rate }\end{array}$ & P-value & Positive & $\begin{array}{l}\text { Positive } \\
\text { rate }\end{array}$ & P-value \\
\hline $\begin{array}{l}\text { Ovarian carcinoma } \\
\text { tissue }\end{array}$ & 60 & 37 & $61.67 \%$ & $<0.01$ & 47 & $78.33 \%$ & $<0.01$ & 43 & $71.67 \%$ & $<0.01$ \\
\hline $\begin{array}{l}\text { Para-carcinoma } \\
\text { normal tissue }\end{array}$ & 60 & 8 & $13.33 \%$ & $<0.01$ & 5 & $8.33 \%$ & $<0.01$ & 7 & $11.67 \%$ & $<0.01$ \\
\hline
\end{tabular}

HMGB1, high mobility group box 1; BRCA1, breast cancer susceptibility gene 1 .

Table III. Drug resistance to cisplatin of 60 ovarian cancer patients.

\begin{tabular}{lcc}
\hline Groups & Case (n) & Ratio (\%) \\
\hline Drug-resistance & 38 & 63.33 \\
Sensitive & 22 & 36.67 \\
\hline
\end{tabular}

ovarian carcinoma and para-carcinoma normal tissues were $71.67 \%(43 / 60)$ and $11.67 \%(7 / 60)$, and the difference was statistically significant $(\mathrm{P}<0.01)$.

Drug resistance to cisplatin of 60 ovarian cancer patients. The results of in vitro resin droplet experiment showed that 38 out of 60 ovarian cancer patients had drug resistance to cisplatin, while 22 cases were sensitive to cisplatin (Table III).

Association of protein expression of $H M G B 1, B R C A 1$ and P62 with drug resistance to cisplatin in ovarian cancer. The results showed that the positive rates of the protein expression of HMGB1, BRCA1 and P62 in 38 patients in the drug resistance group were 84.21, 94.74 and $92.11 \%$, respectively, while those in the sensitive group were 22.73 , 50.00 and $36.36 \%$, respectively. The results of the Chi-square test showed that the positive rates of the protein expression of HMGB1, BRCA1 and P62 in the drug resistance group were significantly higher than those in the sensitive group $(\mathrm{P}<0.01)$ (Table IV).

\section{Discussion}

Clinical study statistics found that the 5-year survival rate of patients with advanced ovarian cancer is lower than $20 \%$, and an important reason leading to poor prognosis is the drug resistance of patients to chemotherapy drugs (11). Therefore, identification of the specific indexes to predict the sensitivity of ovarian cancer patients to chemotherapy drugs are issues to be solved, which can provide powerful assistance for clinical doctors in formulating therapeutic regimens and selecting chemotherapy drugs (12).

Cisplatin, a common chemotherapy drug in clinical tumor therapy, is the first-line chemotherapy drug in ovarian cancer treatment, and drug resistance to cisplatin is the main reason affecting the curative effect of chemotherapy. The drug resistance to cisplatin of ovarian cancer patients can be divided into inherent or acquired drug resistance, and the drug resistance mechanism of tumor is very complex. Currently, the molecular mechanism of patient's drug resistance to cisplatin remains unclear (13).

HMBG1 can inhibit cell apoptosis, and when HMBG1 is overexpressed in cells, tumor cell apoptosis is markedly decreased. When siRNA is used to silence HMGB1 expression, the sensitivity of tumor cells to chemotherapy drugs can be elevated (14). BRCAl gene is located on human chromosome $17 \mathrm{q} 21$, and constitutes the susceptibility gene for breast and ovarian cancer (15). BRCA1 has the effect of inhibiting cell growth and plays a key role in gene transcription, DNA damage repair and apoptosis, especially in maintaining the genome stability (16). P62 is a kind of autophagy-induced protein, and cells can relieve the endoplasmic reticulum stress by autophagy,

Table IV. Association of protein expression of HMGB1, BRCA1 and P62 with drug-resistance to cisplatin in ovarian cancer.

\begin{tabular}{|c|c|c|c|c|c|c|c|c|c|c|}
\hline \multirow[b]{2}{*}{ Groups } & \multirow[b]{2}{*}{ No. } & \multicolumn{3}{|c|}{ HMGB1 } & \multicolumn{3}{|c|}{ BRCA1 } & \multicolumn{3}{|c|}{ P62 } \\
\hline & & Positive & $\begin{array}{c}\text { Positive } \\
\text { rate }\end{array}$ & P-value & Positive & $\begin{array}{c}\text { Positive } \\
\text { rate }\end{array}$ & P-value & Positive & $\begin{array}{l}\text { Positive } \\
\text { rate }\end{array}$ & P-value \\
\hline Drug resistance & 38 & 32 & $84.21 \%$ & $<0.01$ & 36 & $94.74 \%$ & $<0.01$ & 35 & $92.11 \%$ & $<0.01$ \\
\hline Sensitive & 22 & 5 & $22.73 \%$ & $<0.01$ & 11 & $50.00 \%$ & $<0.01$ & 8 & $36.36 \%$ & $<0.01$ \\
\hline
\end{tabular}

HMGB1, high mobility group box 1; BRCA1, breast cancer susceptibility gene 1 . 
thus decreasing the tumor cell sensitivity to cisplatin. Subsequently, when P62 is highly expressed, intracellular autophagy activity is activated, eventually reducing the drug resistance of ovarian carcinoma cells to cisplatin $(17,18)$.

In order to further explore the expression of HMGB1, BRCA1 and P62 in the tumor tissues of ovarian cancer patients and its effect on the drug resistance of ovarian cancer patients to cisplatin, RT-qPCR was performed to detect the mRNA expression of HMGB1, BRCA1 and P62 in the tumor tissues of ovarian cancer patients. The results showed that the mRNA expression levels of HMGB1, BRCA1 and P62 were significantly higher in ovarian carcinoma tissues compared with those in para-carcinoma normal tissues. In addition, immunohistochemical results showed that the positive protein expression rates of HMGB1, BRCA1 and P62 in ovarian carcinoma tissues were $61.67 \%$ (37/60), $76.33 \%$ (47/60) and $71.67 \%$ (43/60), respectively, which were significantly higher than those in para-carcinoma normal tissues. Moreover, the in vitro resin droplet experiment revealed that 38 out of 60 ovarian cancer patients had drug resistance to cisplatin and 22 cases were sensitive to cisplatin. The positive rates of the protein expression of HMGB1, BRCA1 and P62 in the drug resistance group were $84.21,94.74$ and $92.11 \%$, respectively, while those in the sensitive group were $22.73,50.00$ and $36.36 \%$, respectively. Thus, the positive protein expression rates of HMGB1, BRCA1 and P62 in drug resistance were significantly higher than those in the sensitive group.

Wang et al reported that the higher the intracellular expression level of BRCA is, the lower the cell sensitivity to cisplatin will be (19). Liu et al confirmed that after HMGB1 is added into the cell culture fluid, it can induce cells to develop drug resistance to chemotherapy drugs (14). Yu et al found that P62 protein is overexpressed in tissues of ovarian cancer patients who have drug resistance to cisplatin, and it is involved in the formation mechanism of drug resistance of cells to cisplatin (20). This experiment further confirmed the overexpression of HMGB1, BRCA1 and P62 in tumor tissues of ovarian cancer patients, and it was found that the expression of HMGB1, BRCA1 and P62 was associated with the drug resistance of ovarian cancer patients to cisplatin.

In conclusion, the overexpression of HMGB1, BRCA1 and P62 exists in tumor tissues of ovarian cancer patients. Furthermore, its positive expression is associated with chemotherapy sensitivity of ovarian cancer patients. Thus, HMGB1, BRCA1 and P62 may be molecular markers for the prediction of chemotherapy sensitivity of ovarian cancer patients.

\section{Competing interests}

The authors declare that they have no competing interests.

\section{References}

1. Wang Y, Liu P, Qiu L, Sun Y, Zhu M, Gu L, Di W and Duan Y: Toxicity and therapy of cisplatin-loaded EGF modified mPEG-PLGA-PLL nanoparticles for SKOV3 cancer in mice. Biomaterials 34: 4068-4077, 2013.

2. Wu YJ, Neuwelt AJ, Muldoon LL and Neuwelt EA: Acetaminophen enhances cisplatin- and paclitaxel-mediated cytotoxicity to SKOV3 human ovarian carcinoma. Anticancer Res 33: 2391-2400, 2013.
3. Ong PS, Chan SY and Ho PC: Microarray analysis revealed dysregulation of multiple genes associated with chemoresistance to $\mathrm{As}_{2} \mathrm{O}_{3}$ and increased tumor aggressiveness in a newly established arsenic-resistant ovarian cancer cell line, OVCAR-3/AsR. Eur J Pharm Sci 45: 367-378, 2012.

4. Tang D, Kang R, Zeh HJ III and Lotze MT: High-mobility group box 1 and cancer. Biochim Biophys Acta 1799: 131-140, 2010.

5. Wu D, Ding Y, Wang S, Zhang Q and Liu L: Increased expression of high mobility group box 1 (HMGB1) is associated with progression and poor prognosis in human nasopharyngeal carcinoma. J Pathol 216: 167-175, 2008.

6. Beeghly A, Katsaros D, Chen H, Fracchioli S, Zhang Y, Massobrio M, Risch H, Jones B and $\mathrm{Yu} \mathrm{H}$ : Glutathione S-transferase polymorphisms and ovarian cancer treatment and survival. Gynecol Oncol 100: 330-337, 2006.

7. Stengel C, Newman SP, Leese MP, Potter BV, Reed MJ and Purohit A: Class III beta-tubulin expression and in vitro resistance to microtubule targeting agents. Br J Cancer 102: 316-324, 2010.

8. Quinn JE, James CR, Stewart GE, Mulligan JM, White P, Chang GK, Mullan PB, Johnston PG, Wilson RH and Harkin DP: BRCA1 mRNA expression levels predict for overall survival in ovarian cancer after chemotherapy. Clin Cancer Res 13: 7413-7420, 2007.

9. Moscat J, Diaz-Meco MT and Wooten MW: Signal integration and diversification through the p62 scaffold protein. Trends Biochem Sci 32: 95-100, 2007.

10. Kobayashi H, Tanisaka K, Doi O, Kodama K, Higashiyama M, Nakagawa H, Miyake M, Taki T, Hara S, et al: An in vitro chemosensitivity test for solid human tumors using collagen gel droplet embedded cultures. Int J Oncol 11:449-455, 1997.

11. Siegel RL, Miller KD and Jemal A: Cancer statistics, 2015. CA Cancer J Clin 65: 5-29, 2015.

12. Sakamoto M, Kondo A, Kawasaki K, Goto T, Sakamoto H, Miyake K, Koyamatsu Y, Akiya T, Iwabuchi H, Muroya T, et al: Analysis of gene expression profiles associated with cisplatin resistance in human ovarian cancer cell lines and tissues using cDNA microarray. Hum Cell 14: 305-315, 2001.

13. Takano M, Kudo K, Goto T, Yamamoto K, Kita T and Kikuchi Y: Analyses by comparative genomic hybridization of genes relating with cisplatin-resistance in ovarian cancer. Hum Cell 14: 267-271, 2001.

14. Liu L, Yang M, Kang R, Wang Z, Zhao Y, Yu Y, Xie M, Yin X, Livesey KM, Lotze MT, et al: HMGB1-induced autophagy promotes chemotherapy resistance in leukemia cells. Leukemia 25: 23-31, 2011.

15. Miki Y, Swensen J, Shattuck-Eidens D, Futreal PA, Harshman K, Tavtigian S, Liu Q, Cochran C, Bennett LM, Ding W, et al: A strong candidate for the breast and ovarian cancer susceptibility gene BRCA1. Science 266: 66-71, 1994.

16. Garvin AM, Attenhofer-Haner M and Scott RJ: BRCA1 and BRCA 2 mutation analysis in 86 early onset breast/ovarian cancer patients. J Med Genet 34: 990-995, 1997.

17. Greggio E, Lewis PA, van der Brug MP, Ahmad R, Kaganovich A, Ding J, Beilina A, Baker AK and Cookson MR: Mutations in LRRK2/dardarin associated with Parkinson disease are more toxic than equivalent mutations in the homologous kinase LRRK1. J Neurochem 102: 93-102, 2007.

18. Haugarvoll K, Toft M, Ross OA, White LR, Aasly JO and Farrer MJ: Variants in the LRRK1 gene and susceptibility to Parkinson's disease in Norway. Neurosci Lett 416: 299-301, 2007.

19. Wang L, Wei J, Qian X, Yin H, Zhao Y, Yu L, Wang T and Liu B: ERCC1 and BRCA1 mRNA expression levels in metastatic malignant effusions is associated with chemosensitivity to cisplatin and/or docetaxel. BMC Cancer 8: 97, 2008.

20. Yu H, Su J, Xu Y, Kang J, Li H, Zhang L, Yi H, Xiang X, Liu F and Sun L: p62/SQSTM1 involved in cisplatin resistance in human ovarian cancer cells by clearing ubiquitinated proteins. J Eur Cancer 47: 1585-1594, 2011.

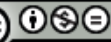

This work is licensed under a Creative Commons Attribution-NonCommercial-NoDerivatives 4.0 International (CC BY-NC-ND 4.0) License. 